\title{
Electric-field ionization of high Rydberg states and vertical ionization potential of an impurity in dense fluid argon
}

\author{
J. Meyer \\ Hamburger Synchrotronstrahlungslabor (HASYLAB) at Deutsches Elektronen-Synchrotron (DESY), 2000 Hamburg 52, Germany \\ R. Reininger \\ Synchrotron Radiation Center, University of Wisconsin-Madison, 3731 Schneider Drive, Stoughton, Wisconsin 53589
}

(Received 4 January 1993)

\begin{abstract}
We present experimental results of field ionization of high Rydberg states of an impurity doped into a dense medium. The experiments allowed us to determine the change of the vertical ionization potential of $\mathrm{CH}_{3} \mathrm{I}$ in fluid argon up to the critical density. The lowering of the vertical ionization potential as a function of the argon number density $(\rho)$ is explained quantitatively on the basis of the energy of the lowest extended state of an excess electron $\left(V_{0}\right)$ in argon and on the sudden polarization energy resulting from the interaction between the dopant ion and the medium. Our results for $V_{0}(\rho)$ are compared with several recent theoretical calculations.
\end{abstract}

PACS number(s): 33.20.Ni, 79.70. $+\mathrm{q}, 33.90 .+\mathrm{h}$

The field ionization of highly excited states has been extensively studied in the case of atomic and molecular states. According to the classical model, a Rydberg state with effective quantum number $n^{*}$ can be ionized by an electric field $F$ (in $\mathrm{V} / \mathrm{cm}$ ), which fulfills [1] $F \geq 3.21 \times 10^{8} / n^{* 4}$. The ionization potential in the presence of the field is, therefore, lowered by $\Delta_{F}=-7.59 \times 10^{-4} \sqrt{F}$, with $\Delta_{F}$ in eV. In this Rapid Communication we present experimental results on electric-field ionization of high Rydberg states of an impurity doped into a dense medium. We find that the lowering of the ionization potential as a function of the applied electric field is proportional to $\sqrt{F}$ in the whole density range investigated. Our motivation for these measurements was to determine the evolution of the vertical ionization potential $\left(I_{p}\right)$ of an impurity doped into a medium as a function of the host density. We should stress here that several studies, performed with either single [2] or multiphonon excitation [3], reported on the density dependence of the photoionization threshold of an impurity doped into several systems. Two investigations performed in low-density rare gases doped with $\mathrm{CH}_{3} \mathrm{I}$ [4] and $\mathrm{C}_{6} \mathrm{H}_{6}$ [5] demonstrated that the energy difference between the vertical ionization potential of the dopant and its photoionization threshold increases significantly with increasing the medium density. Köhler et al. [4] explained this energy difference by a model which assumes that the ionization below $I_{p}$ originates on the photoexcitation of a Rydberg state and on its subsequent ionization induced by medium relaxations.

The model system chosen for this study was methyl iodide doped into varying number densities of Ar. We should emphasize, however, that the results are characteristic of the host rather than the dopant. Their evaluation yields the energy of the lowest extended state of an excess electron $V_{0}$ as a function of the Ar number density $\rho$. Argon was chosen as the host medium since several theoretical approaches based on the Wigner-Seitz model [6-9], on a semiclassical percolation model [10], and on molecular dynamics $[9,11,12]$ have been recently implemented for the calculation of $V_{0}(\rho)$. Most of these calculations showed reasonable agreement when compared to previous experimental results [13]. However, due to the inherent errors involved in the photoinjection experimental technique [13-15], a critical evaluation of the theoretical approaches could not be performed until now.

The experimental setup was described in detail in Ref. [16]. Briefly, monochromatized synchrotron radiation by the HONORMI system at HASYLAB [17] was focused into the center of a high-pressure cell made of stainless steel. The cell was equipped with entrance and exit $\mathrm{LiF}$ windows and a pair of parallel-plate electrodes oriented perpendicular to the windows. The sample temperature was varied between $155 \mathrm{~K}<T<300 \mathrm{~K}$ and the maximum sample pressure was 70 bar. The argon density range covered in the experiments was between the dilute gas and $7.1 \times 10^{21} \mathrm{~cm}^{-3}$. The $\mathrm{CH}_{3} \mathrm{I}$ concentration was in the ppm range. Transmission and photoionization spectra were recorded simultaneously with a resolution of $7 \mathrm{meV}$ and with voltages ranging from a few volts to $3000 \mathrm{~V}$. The corresponding maximum electric field was 7500 $\mathrm{V} / \mathrm{cm}$. Both the photocurrent and transmission spectra were normalized to the incoming photon flux. As described below, the evaluation of the data involved the subtraction of the photocurrent spectra recorded at different electric fields. In order to avoid spurious effects, only the spectra recorded with electric fields exhibiting current saturation in the spectral range above the ionization limit were used in the evaluation.

Figure 1 shows, as an example, photocurrent spectra of $\mathrm{CH}_{3} \mathrm{I}$ doped into argon at a density of $4.6 \times 10^{21} \mathrm{~cm}^{-3}$ for several applied electric fields. As seen in the figure the 


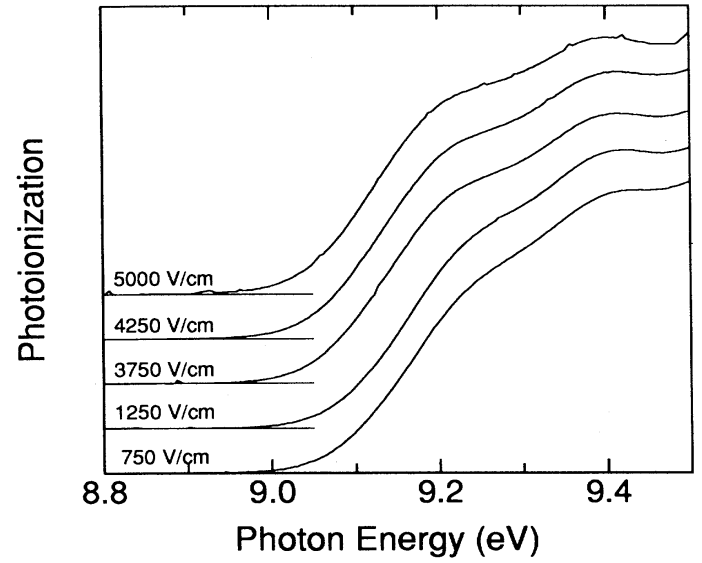

FIG. 1. Photoionization spectra of $\mathrm{CH}_{3} \mathrm{I}$ doped into argon for several applied electric fields. The sample density and pressure are $4.6 \times 10^{21} \mathrm{~cm}^{-3}$ and $171 \mathrm{~K}$, respectively.

photoionization threshold shifts from $\approx 8.96$ to $\approx 8.92$ $\mathrm{eV}$ with an increase in the electric field. Furthermore, there is an increase in the photocurrent signal below 9.3 $\mathrm{eV}$. A clear visualization of this effect is presented in Fig. 2(a). These spectra were obtained from those displayed in Fig. 1 by subtracting the spectrum recorded at $750 \mathrm{~V} / \mathrm{cm}$ from those measured at higher fields. As Fig. 2(a) demonstrates there is a significant increase in the current between 8.93 and $9.31 \mathrm{eV}$ with an increase in the electric field. Figure 2(b) displays similar results obtained by subtracting the spectrum recorded at $3750 \mathrm{~V} / \mathrm{cm}$ from those measured at 4250 and $5000 \mathrm{~V} / \mathrm{cm}$. As mentioned above, Köhler et al. [4] attributed the current below $I_{p}$ to the ionization of Rydberg states induced by medium relaxations. The shift of the photoionization threshold shown

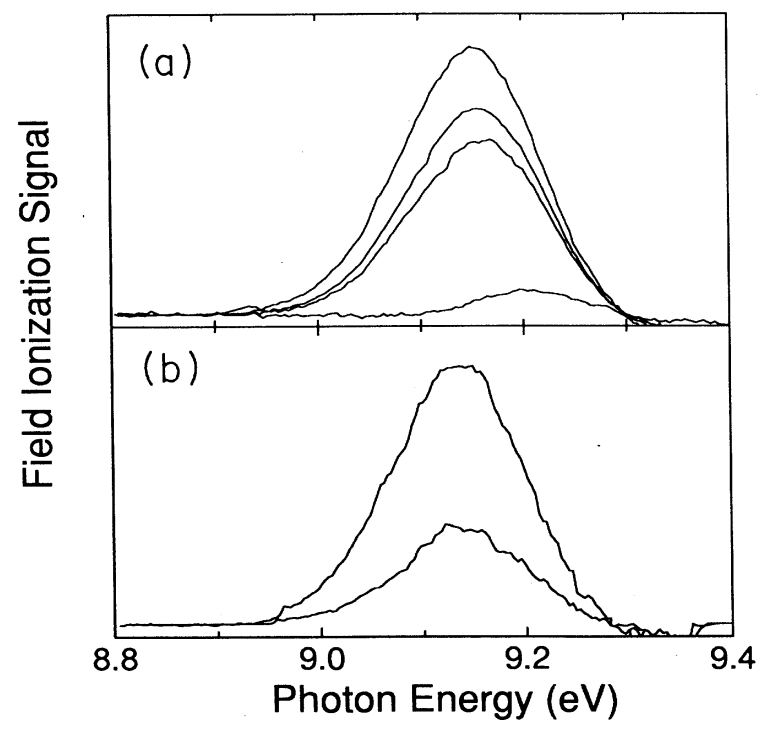

FIG. 2. Electric-field ionization signal of $\mathrm{CH}_{3} \mathrm{I}$ doped into argon as a function of the photon energy. The spectra were obtained from the results shown in Fig. 1 by subtracting the spectra recorded at (a) $750 \mathrm{~V} / \mathrm{cm}$ and (b) $3750 \mathrm{~V} / \mathrm{cm}$ from those measured at higher electric fields. in Fig. 1 and the field ionization signal displayed in Fig. 2 can be explained in terms of this model [4] and by incorporating the medium effects on the model for classical field ionization [1]; i.e., the applied electric field lowers the ionization potential in the media by $\Delta_{F}=-7.59 \times 10^{-4} \sqrt{F} / \sqrt{\epsilon}$ ( $\epsilon$ is the medium dielectric constant), and consequently, increases the probability of ionizing the photoexcited Rydberg states. Since the applied electric field should not induce additional current above the ionization limit, the high-energy intercept of the curves shown in Fig. 2(a) (at 9.31 10.01 ) and 2(b) (at $9.28 \pm 0.01 \mathrm{eV}$ ) corresponds to the ionization potential of $\mathrm{CH}_{3} \mathrm{I}$ in the sample at 750 and $3750 \mathrm{~V} / \mathrm{cm}$, respectively. It should be mentioned that the high Rydberg states responsible for the additional current signal are not resolved in the absorption or ionization spectra due to the high density of the sample.

Using the same subtraction procedure, we determined the ionization potential for the different applied electric fields. These results and their corresponding errors are displayed in Fig. 3 as a function of the square root of the electric field for several argon densities. The lines shown in the figure represent linear fits to the data, taking into account the error in the determination of each data point. The intercepts of the lines with the abscissa determine the vertical ionization potential (IP) of $\mathrm{CH}_{3} \mathrm{I}$ at the given argon density. The slopes of the fitted lines vary between $-0.83 \mathrm{meV} /(\mathrm{V} / \mathrm{cm})^{1 / 2}$ at low argon densities to -0.71 $\mathrm{meV} /(\mathrm{V} / \mathrm{cm})^{1 / 2}$ at higher densities. We note that the change in the dielectric constant yields an increase in the slope from -0.76 to $-0.68 \mathrm{meV} /(\mathrm{V} / \mathrm{cm})^{1 / 2}$ in the same density range.

The full circles in Fig. 4(a) summarize our results for the density dependence of the vertical ionization potential of $\mathrm{CH}_{3} \mathrm{I}$ in argon. The error bars represent the uncertainty in the determination of the intercept as given by the linear fit procedure. We remark that the vertical ionization limit for $\rho=4.6 \times 10^{21} \mathrm{~cm}^{-3}$ is $9.33 \pm 0.02 \mathrm{eV}$, i.e., at least $0.4 \mathrm{eV}$ larger than the ionization thresholds

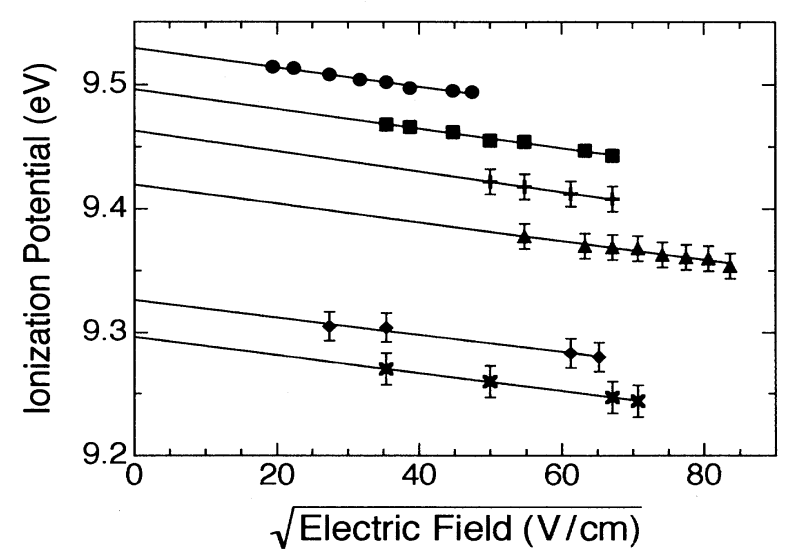

FIG. 3. Ionization potential of $\mathrm{CH}_{3} \mathrm{I}$ doped into varying argon densities as a function of the square root of the electric field. The number densities of argon are, $1.5 \times 10^{20} \mathrm{~cm}^{-3} ; \mathbf{\square}$, $8.2 \times 10^{20} \mathrm{~cm}^{-3} ;+, 15.1 \times 10^{20} \mathrm{~cm}^{-3} ; \boldsymbol{\Delta}, 23.2 \times 10^{20} \mathrm{~cm}^{-3} ; \boldsymbol{\nabla}$, $46 \times 10^{20} \mathrm{~cm}^{-3}$; and $\times, 52 \times 10^{20} \mathrm{~cm}^{-3}$. 


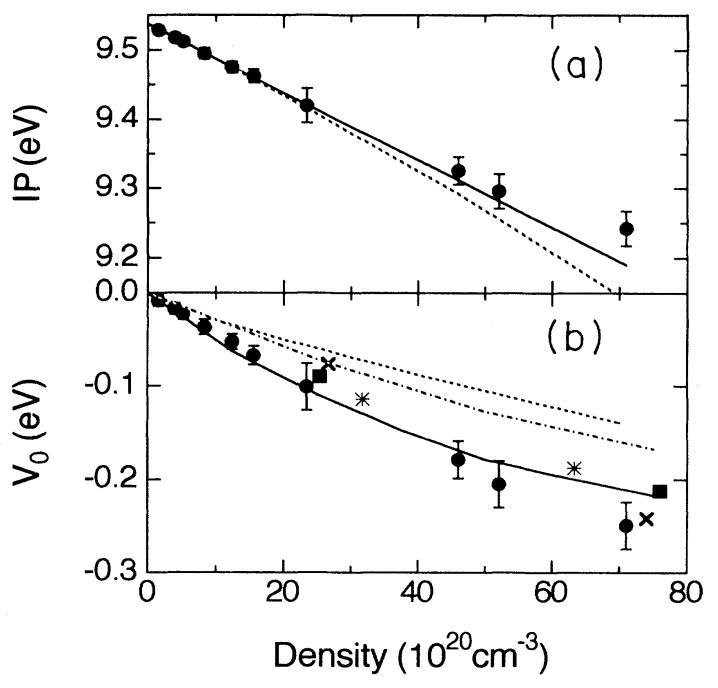

FIG. 4. (a) Vertical ionization potential of $\mathrm{CH}_{3} \mathrm{I}$ as a function of the Ar number density. present results; solid line, from Refs. [16,18]; dashed lines, extrapolation of the modified Fermi model at the thermodynamical conditions of our data. (b) Density dependence of the lowest extended state of an electron in Ar. $\bigcirc$, present results; $-\cdot-\cdot-\cdot,-\_, \cdots . \times, \times, \mathbf{\square}$, and $*$, theoretical calculations from Refs. [8], [9], [10], [9], [11], and [12], respectively.

shown in Fig. 1. The solid line included in the figure depicts the linear dependence of the vertical ionization potential as obtained from the shift of the high-energy Rydberg states of $\mathrm{CH}_{3} \mathrm{I}$ doped into room-temperature argon $[16,18]$. These results $[16,18]$ were explained in terms of a modified Fermi model $[4,19,20]$ stating that the shift of the high Rydberg states and, consequently, the shift of the ionization potential are given by

$$
\Delta=\left(2 \pi \hbar^{2} / m\right) a \rho-6.79\left(\alpha e^{2}\right)^{2 / 3}(\hbar v)^{1 / 3} \rho,
$$

where $\hbar$ is the Planck constant, $m$ and $e$ are the electron mass and charge, respectively, $a$ is the electron-perturber scattering length, $\alpha$ is the perturber polarizability, and $v$ is the relative velocity of the colliding partners. The first term in Eq. (1) is due to the interaction of the electron with the medium; the second term is due to the sudden polarization of the medium by the positive core. We note that only the host atoms situated outside the Weisskopf sphere, i.e., a sphere with radius $\left(\pi \alpha e^{2} / 4 \hbar v\right)^{1 / 3}$ and centered at the positive core, contribute to the polarization term $[4,20]$. As seen in the figure the two data sets are in perfect agreement in the common density range, i.e., up to $\rho=1.2 \times 10^{21} \mathrm{~cm}^{-3}$, further corroborating our method and the interpretation of our results. It should be stressed at this point that the vertical ionization potential of an impurity cannot be determined by pressure shift studies on Rydberg states at argon densities larger than those reported in Ref. [18] due to the large broadening of the Rydberg states.

The dotted line in Fig. 4(a) shows the evaluation of the vertical ionization potential according to Eq. (1) at the thermodynamical conditions of our experimental data. Evidently, the calculation does not agree with our experi- mental results above $2.4 \times 10^{21} \mathrm{~cm}^{-3}$. The reason for this disagreement is obvious; Eq. (1) was derived for the lowdensity regime, and consequently neglects the contribution of repulsive interactions, which become significant at high densities.

The repulsive interactions can be readily included by assuming that the energy shift of the vertical ionization potential at high densities can be separated into the contributions due to the positive ion and due to the electron, as in Eq. (1). The repulsive interactions between the induced dipoles can be taken into account by including a screening function [21] in the second term of Eq. (1). Since in the density range covered in the present experiments the Weisskopf radius is larger than $1.8 \mathrm{~nm}$, the screening function can be replaced by the Lorentz local field correction for a structureless fluid [22]

$$
f_{\infty}=(1+8 \pi \rho \alpha / 3)^{-1} .
$$

The interaction between the electron and the medium for all densities is described, in fact, by $V_{0}(\rho)$, the energy of the lowest extended state of an excess electron in the medium. We note in passing that the first term appearing in Eq. (1) is the low-density limit of $V_{0}$.

Based on the above considerations, we can express the shift of the vertical ionization potential as

$$
\Delta^{\prime}=V_{0}-6.79\left(\alpha e^{2}\right)^{2 / 3}(\hbar v)^{1 / 3} \rho f_{\infty} .
$$

It is worth mentioning at this point that the polarization term in Eq. (3) is much smaller than the adiabatic polarization energy given by Messing and Jortner [22].

Figure 4(b) displays the density dependence of $V_{0}$ in $\mathrm{Ar}$ obtained by combining our experimental results for the shift of the vertical ionization potential and Eq. (3). We included in the figure the most recent calculations of $V_{0}(\rho)$ performed by Lopez-Castillo et al. [9] using both the Wigner-Seitz model and path-integral molecular dynamics, and those of Stampfli and Bennemann [8], Simon, Dobrosavljevic, and Stratt [10], Space et al. [11], and Boltjes, de Graaf, and de Leeuw [12]. As seen in the figure our results for $\rho \leq 4.6 \times 10^{21} \mathrm{~cm}^{-3}$ are in good agreement with the theoretical calculations performed by Lopez-Castillo et al. [9] based on the Wigner-Seitz model (which does not take into account the fluidlike character of the solvent) and using the electron-argon pseudopotential of Li, Broughton, and Allen [23]. The agreement between our results and their calculations for the two highest densities is, however, less satisfactory. Stampfli and Bennemann performed a Wigner-Seitz-type calculation approximating the effect of decreasing density as being due to an increase in the number of vacant lattice sites. They introduced, however, a very simple electronargon pseudopotential. Their results [8] are consistently higher than our data. This is also the case with the calculations performed by Simon, Dobrosavljevic, and Stratt [10] in terms of a semiclassical percolation model that takes into account the fluid disorder but ignores manybody polarization interactions. Three groups $[9,11,12]$ recently calculated $V_{0}$ using quantum molecular dynamics. They demonstrated $[9,11,12]$ that $V_{0}$ shows the experimentally observed minimum [13] only when the in- 
teractions between the induced dipoles are properly taken into account. Due to the long computational times involved in this type of calculation all the groups computed only two densities in the range of our experimental results. Lopez-Castillo et al. [9] used in their calculations the electron-atom potential of $\mathrm{Li}$, Broughton, and Allen [23] and a simple form for the screening function. As shown in Fig. 4(b), their [9] high-density result is in good agreement with our data. Space et al. [11] employed a self-consistent form for the screening function and a parametrized pseudopotential. Using a similar screening function, Boltjes, de Graaf, and de Leeuw [12] showed the insensitivity of their low-density results to the choice of the pseudopotential. As seen in the figure, only the low-density results of both groups $[11,12]$ agree with our data.

In summary, we have shown that field ionization can be exploited to determine the vertical ionization potential of an impurity doped into a dense medium. From these results we were able to determine the energy of the lowest extended state of an excess electron in argon up to almost the critical density.

We would like to thank Dr. B. Plenkiewicz for providing us with her recent calculations. This work was supported, in part, by the National Science Foundation.
[1] H. A. Bethe and E. E. Salpeter, Quantum Mechanics of One- and Two-Electron Atoms (Academic, New York, 1957).

[2] See, e.g., I. T. Steinberger, in The Liquid State and Its Electrical Properties, edited by E. E. Kunhardt, L. G. Christophorou, and L. H. Huessen (Plenum, New York, 1988), p. 235.

[3] H. Faidas, L. G. Christophorou, P. G. Datskos, and D. L. McCorkle, J. Chem. Phys. 90, 6619 (1989).

[4] A. M. Köhler, V. Saile, R. Reininger, and G. L. Findley, Phys. Rev. Lett. 60, 2727 (1988).

[5] R. Reininger, E. Morikawa, and V. Saile, Chem. Phys. Lett. 159, 276 (1989).

[6] B. Plenkiewicz, J. P. Jay-Gerin, P. Plenkiewicz, and G. B. Bachelet, Europhys. Lett. 1, 455 (1986).

[7] B. Plenkiewicz, P. Plenkiewicz, and J.-P. Jay-Gerin, Phys. Rev. A 40, 4113 (1989).

[8] P. Stampfli and K. H. Bennemann, Phys. Rev. A 44, 8210 (1991).

[9] J.-M. Lopez-Castillo, Y. Frongillo, B. Plenkiewicz, and J.-P. Jay-Gerin, J. Chem. Phys. 96, 9092 (1992).

[10] S. H. Simon, V. Dobrosavljevic, and R. M. Stratt, J. Chem. Phys. 94, 7360 (1991).

[11] B. Space, D. F. Coker, Z. H. Liu, B. J. Berne, and G. Mar- tyna, J. Chem. Phys. 97, 2002 (1992).

[12] B. Boltjes, C. de Graaf, and S. W. de Leeuw, J. Chem. Phys. 98, 592 (1993).

[13] R. Reininger, U. Asaf, I. T. Steinberger, and S. Basak, Phys. Rev. B 28, 4426 (1983).

[14] R. Reininger, U. Asaf, and I. T. Steinberger, Chem. Phys. Lett. 90, 287 (1982).

[15] W. F. Schmidt, in Excess Electrons in Dielectric Media, edited by C. Ferradini and J.-P. J. Gerin (CRC, Boca Raton, FL, 1991), p. 127.

[16] J. Meyer, R. Reininger, and U. Asaf, Chem. Phys. Lett. 173, 384 (1990).

[17] V. Saile, P. Gürtler, E. E. Koch, A. Kozevnikov, M. Skibowski, and W. Steinmann, Appl. Opt. 15, 2559 (1976).

[18] A. M. Köhler, R. Reininger, V. Saile, and G. L. Findley, Phys. Rev. A 33, 771 (1986).

[19] E. Fermi, Nuovo Cimento 11, 157 (1934).

[20] V. A. Alekseev and I. I. Sobel'mann, Zh. Eksp. Teor. Fiz. 49, 1274 (1965) [Sov. Phys. JETP 22, 882 (1966)]

[21] J. Lekner, Phys. Rev. 158, 130 (1967).

[22] I. Messing and J. Jortner, Chem. Phys. 24, 183 (1977).

[23] X.-P. Li, J. Q. Broughton, and P. B. Allen, J. Chem. Phys. 85, 3444 (1986). 